\title{
Einfluss gestufter Lernhilfen als Unterstützungsmaßnahme beim Experimentieren auf den Lernerfolg im Biologieunterricht
}

\author{
Cornelia Stiller (D) Matthias Wilde
}

Eingegangen: 24. März 2020 / Überarbeitet: 21. Dezember 2020 / Angenommen: 26. März 2021 / Online publiziert: 19. April 2021

(C) Der/die Autor(en) 2021

Zusammenfassung Problemlöseaufgaben wie naturwissenschaftliche Schülerexperimente können von Lernenden oft nicht ohne Unterstützung bearbeitet werden. Diese Unterstützung können gestufte Lernhilfen bieten. In der vorliegenden Studie wurde untersucht, inwiefern gestufte Lernhilfen beim Experimentieren Auswirkungen auf den Wissenszuwachs haben und inwiefern der Wissenszuwachs vom Vorwissen beeinflusst wird. Lernende der Experimentalgruppe (EG) arbeiteten während des Experimentierens mit gestuften Lernhilfen, während die Kontrollgruppe (KG) keine zusätzlichen Unterstützungsmaßnahmen erhielt. Die Ergebnisse zeigen, dass sich beide Gruppen hinsichtlich des prozeduralen Wissenszuwachs zugunsten der EG signifikant unterschieden, nicht aber bezüglich des konzeptuellen Wissenszuwachses. Sowohl konzeptuelles als auch prozedurales Vorwissen konnten als Prädiktor für konzeptuelles bzw. prozedurales Wissen nach dem Unterricht identifiziert werden. Zusätzlich erwies sich das Treatment als Prädiktor für das prozedurale Wissen im Nachtest, interagierte aber nicht signifikant mit dem Vorwissen. Es ist demnach davon auszugehen, dass Lerner unabhängig vom Vorwissen von den gestuften Lernhilfen profitieren können.

Schlüsselwörter Experimentieren · Gestufte Lernhilfen · Naturwissenschaftliche Erkenntnisgewinnung $\cdot$ Vorwissen

\footnotetext{
C. Stiller

Fakultät für Biologie/Biologiedidaktik und Fakultät für Erziehungswissenschaften/AG4, Universität Bielefeld, Universitätsstraße 25, 33615 Bielefeld, Deutschland

E-Mail: cornelia.stiller@uni-bielefeld.de

Prof. Dr. M. Wilde $(\bowtie)$

Fakultät für Biologie/Biologiedidaktik, Universität Bielefeld, Universitätsstraße 25, 33615 Bielefeld, Deutschland

E-Mail: matthias.wilde@uni-bielefeld.de
} 


\title{
Influence of incremental scaffolds during experimenting on learning success in biology lessons
}

\begin{abstract}
Experiments in science lessons are considered problem-solving tasks. Students are often unable to cope with these tasks without support such as incremental scaffolds. Our study is based on the following research question: Do incremental scaffolds during experimentation have positive effects on conceptual and procedural knowledge acquisition? We also investigated, whether the knowledge in the posttest was determined by prior knowledge. The experimental group worked with incremental scaffolds during experimentation. The control group received no additional support. In conceptual knowledge acquisition no difference between both groups was found. In procedural knowledge the experimental group benefitted. Conceptual prior knowledge was found to be a predictor for conceptual knowledge in posttest. Procedural prior knowledge and treatment were found to be predictors for procedural knowledge in posttest. Treatment and prior knowledge do not interact significantly with each other. Incremental scaffolds apparently promote procedural knowledge regardless of procedural prior knowledge.
\end{abstract}

Keywords Experimentation · Incremental scaffolds $\cdot$ Prior knowledge $\cdot$ Scientific inquiry

\section{Einleitung}

Die Fähigkeit zum Experimentieren ist in den naturwissenschaftlichen Lehrplänen in NRW im Kompetenzbereich Erkenntnisgewinnung verankert (z. B. für Biologie: MSW NRW 2013) und stellt ein festgeschriebenes Lernziel für die Mittelstufe sowie einen wesentlichen Bestandteil der nationalen Bildungsstandards zur Erlangung einer naturwissenschaftlichen Grundbildung dar (KMK 2005). Lernenden konnten in internationalen Vergleichsstudien Defizite im Bereich der Teilfähigkeiten naturwissenschaftlicher Erkenntnisgewinnung nachgewiesen werden (Hammann und Prenzel 2008; OECD 2007; Schiepe-Tiska et al. 2016). Darüber hinaus können Anforderungen durch naturwissenschaftliche Problemlöseaufgaben, wie sie beim Experimentieren an die Lernenden herangetragen werden, zu einer Überforderung führen (Schmidt-Weigand et al. 2008), gerade wenn diese ein unterschiedliches Vorwissen aufweisen (Stäudel et al. 2007). Bei einer Aufgabenbearbeitung sind Lernende je nach Lernausgangslage auf mehr oder weniger Unterstützung angewiesen (Abels 2015), die den Experimentierprozess der Lernenden strukturiert (Dohn 2013; Krajcik et al. 1998). Dabei sollte die Unterstützung an das Vorwissen und die Experimentierfähigkeiten der Lernenden angepasst werden (de Jong 2005). Häufig basieren Maßnahmen zur Unterstützung des Experimentierprozesses auf der Bearbeitung stark vorstrukturierter Anleitungen. Die Eignung derartiger Anleitungen wird allerdings hinterfragt (Sadeh und Zion 2012), zumal in diesem Zusammenhang meist äußere Aktivitäten mit dem Material und weniger das gedankliche Durchdringen des Experimentierprozesses fokussiert werden (Hodson 1998; Hofstein und Lunetta 2004). Experimentieren allein genügt nicht, um ein Verständnis des naturwissenschaftli- 
chen Erkenntnisprozesses zu erwerben, sondern den Lernenden muss explizit die Möglichkeit gegeben werden, den Experimentierprozesses zu reflektieren (Bell et al. 2003). Ein Unterstützungsformat, das zwischen instruktionaler Anleitung und Nachdenken beim Aufgabenlösen im naturwissenschaftlichen Unterricht vermitteln kann, stellen gestufte Lernhilfen dar (z. B. Schmidt-Weigand et al. 2008). Durch gestufte Lernhilfen kann die Komplexität von Aufgaben und damit die Anforderungen der Bearbeitung für die Lernenden reduziert werden (Hänze et al. 2007). Gestufte Lernhilfen können demnach eine dem Bedarf der jeweiligen Schüler*innen angepasste Unterstützung ermöglichen (Hänze et al. 2010). Dabei können gestufte Lernhilfen insbesondere leistungsschwächere Lernende unterstützen (Schmidt-Weigand et al. 2008).

In Studien zur Wirksamkeit von gestuften Lernhilfen bei der Bearbeitung von naturwissenschaftlichen Problemen im Chemieunterricht zeigte sich ein positiver Effekt der Lernhilfen für den Lernerfolg der Schüler*innen (Schmidt-Weigand et al. 2008). Bisherige Untersuchungen zur Unterstützung der einzelnen Teilprozesse beim Experimentieren im Biologieunterricht bei Schüler*innen der Sekundarstufe II durch gestufte Lernhilfen zeigen, bezogen auf Wissen, keinen Vorteil gegenüber Lernenden, die keine zusätzliche Unterstützung erhalten haben (Arnold et al. 2017). Weitere Untersuchungen zur Wirksamkeit von gestuften Lernhilfen, insbesondere für die Unterstützung der einzelnen Teilprozesse beim naturwissenschaftlichen Experimentieren jüngerer Lernenden liegen zurzeit nicht vor.

Vor diesem Hintergrund soll in dieser Studie die Wirksamkeit gestufter Lernhilfen für das Experimentieren im Biologieunterricht, die die einzelnen Teilprozesse des Experimentierens unterstützen, hinsichtlich des Wissenserwerbs untersucht werden. Insbesondere steht im Fokus dieser Untersuchung, inwieweit gestufte Lernhilfen, eine Unterstützung von Lernenden in wissensheterogenen Lerngruppen beim naturwissenschaftlichen Experimentieren ermöglichen.

\section{Theorie und Forschungsstand}

\subsection{Naturwissenschaftliche Erkenntnisgewinnung im experimentellen Biologieunterricht}

Naturwissenschaftliche Denk- und Arbeitsweisen (Scientific Inquiry) stehen für das grundlegende Prinzip moderner angewandter Naturwissenschaften (Colburn 2000; Flick und Lederman 2006). Experimentieren im naturwissenschaftlichen Unterricht kann dazu beitragen, ein Verständnis für den Prozess der naturwissenschaftlichen Denk- und Arbeitsweisen zu erlangen (Wirth et al. 2008). Scientific Inquiry im naturwissenschaftlichen Unterricht beschreibt einen forschend-entdeckenden Ansatz, bei dem Lernende die Perspektive von Naturwissenschaftlerinnen und Naturwissenschaftlern einnehmen und ein Verständnis von naturwissenschaftlicher Erkenntnisgewinnung entwickeln sowie Wissen über naturwissenschaftliche Phänomene erwerben sollen (Colburn 2000; Flick und Lederman 2006; NRC 2012). Der Prozess naturwissenschaftlicher Erkenntnisgewinnung kann als ein kognitiver Problemlöseprozess charakterisiert werden (Abd-El-Khalick et al. 2004; Gott und Roberts 
2008; Klahr 2000), der zentrale konzeptuelle und prozedurale Anforderungen an die Lernenden stellt (Zimmerman 2005). Unter konzeptuellem Wissen wird das domänenspezifische Wissen über Fakten, Konzepte, Gesetze, Theorien (Glaesser et al. 2009; Gott und Roberts 2008) und Prinzipien (de Jong und Ferguson-Hessler 1996; Furtak et al. 2012) verstanden, das zur Aufgabenbearbeitung herangezogen wird (de Jong und Ferguson-Hessler 1996). Erfahrungen mit naturwissenschaftlichen Phänomenen werden für den Erwerb konzeptuellen Wissens als wesentlich herausgestellt (Kollöffel und de Jong 2013; Papadouris und Constantinou 2009). Prozedurales Wissen wird als Subdimension des epistemischen Wissens, dem Wissen über den Prozess naturwissenschaftlicher Erkenntnisgewinnung, beschrieben (Furtak et al. 2012). Es nimmt vor diesem Hintergrund Bezug auf das Verständnis für die prozessbezogenen, wissenschaftsmethodischen Teilfähigkeiten der naturwissenschaftlichen Erkenntnisgewinnung und beinhaltet Fähigkeiten, Techniken oder Methoden, die subjekt- oder disziplinspezifisch sind (Anderson et al. 2001). Für den naturwissenschaftlichen Erkenntnisprozess werden u. a. das Formulieren naturwissenschaftlicher Fragestellungen, das Generieren von Hypothesen, das Planen und Durchführen von Untersuchungen, die Analyse der Daten und das Schlussfolgern als prozessbezogene Teilfähigkeiten benannt (z. B. Abd-El-Khalick et al. 2004; Furtak et al. 2012; KMK 2005; NRC 2012; Zimmerman 2005).

Das Experimentieren stellt eine charakteristische fachgemäße Arbeitsweise zur Lösung naturwissenschaftlicher Probleme im Sinne des naturwissenschaftlichen Erkenntnisgewinns dar (Hammann et al. 2007; Klahr 2000; Osborne et al. 2003). Experimente durchführen zu können und zu verstehen, gilt als ein zentrales Ziel naturwissenschaftlichen Unterrichts (KMK 2005; Osborne et al. 2003; Wirth et al. 2008). In diversen Studien wurden Lernenden allerdings Defizite bezüglich der experimentellen Methode nachgewiesen. So zeigen sie Schwierigkeiten beim Aufstellen von Hypothesen, der systematischen Planung und Durchführung von Experimenten und der Dateninterpretation (zusammengefasst nach de Jong und van Joolingen 1998). Um das Potenzial von Experimenten für das Lernen nutzen zu können, wird die Implementation einer angemessenen instruktionalen Unterstützung vorgeschlagen (Mayer 2004; Wirth et al. 2008; de Jong 2019).

\subsection{Instruktionale Unterstützung und Vorwissen}

Interaktive Lernumgebungen, wie z. B. beim Experimentieren, stellen hohe Anforderungen an die Lernenden (Wirth et al. 2008). Das Lösen naturwissenschaftlicher Probleme erfordert neben dem konzeptuellen Lernen zusätzliche Ressourcen, wie die Anwendung von Problemlösestrategien (Kirschner et al. 2006; Paas et al. 2003). Zudem sollen die Lernenden kognitive Strukturen möglichst selbstständig aufbauen (Einsiedler und Hardy 2010). Allerdings erwies sich in bisherigen Untersuchungen vollständig unangeleitetes Experimentieren als nicht wirksam (Lazonder und Harmsen 2016) und es wird empfohlen, den Experimentierprozess durch direkte Instruktionen zu begleiten oder eine direkte Instruktion dem selbstständigen Experimentieren voranzustellen (de Jong 2019). Ist der Anteil selbstständiger Erarbeitung zu hoch, ist es für Lernende schwierig, die kognitiven Anforderungen zu bewältigen (Einsiedler und Hardy 2010; Hardy et al. 2006; Kalyuga 2013; Mayer 2004). 
Die Komplexität des naturwissenschaftlichen Problemlösens kann durch eine Erhöhung des Anteils instruktionaler Anleitung reguliert werden (vgl. Blanchard et al. 2010; Sadeh und Zion 2012). Ein Vorteil instruktionaler, unterstützender Maßnahmen gegenüber dem selbstständigen, offenen Problemlösen konnte hinsichtlich der Lernwirksamkeit bereits gezeigt werden (z. B. Arnold et al. 2014, 2017; Einsiedler und Hardy 2010; Hmelo-Silver et al. 2007; Kirschner et al. 2006; Lazonder und Harmsen 2016; Mayer 2004).

Als Maßnahme instruktionaler Unterstützung zur konstruktiven Beschäftigung mit dem Lerngegenstand wird der gezielte Einsatz von Strukturierungshilfen vorgeschlagen (Hardy 2012; Hardy et al. 2006). Strukturierende Maßnahmen sollen Lernende bei der Verknüpfung von neuen Wissenselementen bzw. bei der Modifikation bereits vorhandener Konzepte unterstützen (Hardy et al. 2006) und Anforderungen von Lernsituationen derart anpassen, dass Lernende ihnen gerecht werden können (Hardy et al. 2006; Kleickmann et al. 2010). Die Strukturierung einer Aufgabe kann ihre Komplexität beeinflussen und so den Schwierigkeitsgrad einer Aufgabe bestimmen (Kalyuga et al. 2001). Die durch strukturierende Maßnahmen zur Verfügung stehenden kognitiven Ressourcen können für den eigentlichen Lernprozess, der die Bearbeitung und Lösung der Aufgabe beinhaltet, aufgebracht werden (SchmidtWeigand et al. 2008). Anhand einer steigenden Spezifität lassen sich strukturierende Maßnahmen in process constraints (Umfang der Lernaufgabe wird beschränkt), performance dashboards/status overviews (direkte Rückmeldung an die Lernenden über den Stand im Lernprozess), Prompts (Hinweise, eine bestimmte Handlung durchzuführen), Heuristiken (Anregungen dazu, eine Handlung auf eine bestimmte Weise auszuführen), Scaffolds (Erläuterungen zu komplexeren Teilen des Lernprozesses) und Erklärungen (Präsentation von konkreten Informationen wie eine Lernhandlung auszuführen ist) differenzieren (de Jong und Lazonder 2014; Lazonder und Harmsen 2016). Diese Unterstützungsmaßnahmen wurden zwar für computergestütztes Lernen beschrieben, lassen sich aber auf „reale“ forschende Lernsituationen übertragen.

Die Komplexität einer Aufgabe wird neben ihrer Strukturierung auch durch das Vorwissen der Lernenden beeinflusst (Kalyuga et al. 2001; Sweller et al. 1998). Darüber hinaus gilt das Vorwissen als wesentlicher Bedingungsfaktor für den Lernerfolg (Kalyuga 2013; Kalyuga et al. 2001; Kirschner et al. 2006). Verfügen Lernende in einem spezifischen Inhaltsbereich über ein hohes Vorwissen, kann ein neuer Sachverhalt mit geringerem kognitivem Aufwand verarbeitet werden als von Lernenden mit wenig Vorwissen. Lernende mit geringem Vorwissen fühlen sich insbesondere bei komplexen Aufgaben eher überfordert (Einsiedler und Hardy 2010; Kalyuga 2007, 2013; Sweller et al. 1998). Eine typische Reaktion auf dieses Problem ist oft, die Komplexität einer Aufgabe für alle Lernenden einer Klasse in gleichem Maße zu reduzieren, indem die Aufgabe so strukturiert wird, dass ein mittleres Anforderungsniveau adressiert wird (Hänze et al. 2007).

Das erforderliche Maß instruktionaler Unterstützung variiert allerdings je nach Vorwissensstand der Lernenden. So profitieren insbesondere Lernende mit einem geringen Vorwissen von zusätzlichen unterstützenden Maßnahmen, während Lernende mit mehr Vorwissen Aufgaben bewältigen können, die in ihrer Komplexität nicht reduziert wurden (Einsiedler und Hardy 2010; Kalyuga 2007; Kalyuga et al. 2003; Kirschner et al. 2006; Sweller et al. 1998). Die Aufgabengestaltung für das 
naturwissenschaftliche Problemlösen sollte passend an die individuellen Voraussetzungen der Lernenden adaptiert werden, um ein angemessenes Verhältnis zwischen Anleitung und eigenständigem Erkunden zu erreichen (Mayer 2004). Instruktionale Designs, die eine optimale Passung zwischen den jeweiligen Schülerfähigkeiten und Aufgabenanforderungen anstreben (Helmke 2015), sollten daher unterschiedliche Vorwissensstände von Lernenden berücksichtigen (Kalyuga 2013; Kalyuga et al. 2001).

\subsection{Aufgaben mit gestuften Lernhilfen}

Gestufte Lernhilfen (Leisen 1999) bieten eine instruktionale Unterstützung bei der Bearbeitung komplexer Aufgaben- oder Problemstellungen und können gleichzeitig an das eigenständige Bearbeiten von Aufgaben heranführen (Hänze et al. 2007). Sie bestehen aus aufeinander aufbauenden, schriftlich formulierten Lösungshinweisen mit Lösungsschritten zu einer Aufgabe, auf die die Lernenden optional während der Aufgabenbearbeitung zurückgreifen können (Schmidt-Weigand et al. 2008, 2009). Nach Schmidt-Weigand et al. (2008) sind gestufte Lernhilfen zweiteilig aufgebaut. Der erste Teil jeder Lernhilfe beinhaltet eine Aufforderung zu einer lernrelevanten, kognitiven Handlung (Prompt). Diese Handlungsaufforderungen orientieren sich an allgemeinen Strategien zum Lernen und Problemlösen und beinhalten unter anderem das Paraphrasieren, Fokussieren, Aktivieren von Vorwissen, Elaborieren von Unterzielen und das Visualisieren (Schmidt-Weigand et al. 2008). Der zweite Teil einer Lernhilfe hält die jeweilige Teillösung bzw. eine inhaltliche Erläuterung bereit und dient den Lernenden als Rückmeldung (Schmidt-Weigand et al. 2008, 2009). Durch die Bereitstellung von Musterlösungen nach dem Denkimpuls soll ein vollständiges Durchlaufen des Problemlöseprozesses sichergestellt werden (Franke-Braun et al. 2008). Prompts dienen dabei nicht ausschließlich der Reduktion von Komplexität, sondern fokussieren auf wesentliche Aspekte der Aufgabe und sollen eine Aufgabenbearbeitung ohne gedankliche Beteiligung verhindern (Hmelo-Silver et al. 2007). Im Falle der gestuften Lernhilfen wird durch den Einsatz der vorgeschalteten Denkimpulse (Prompts) einer bloßen Rezeption der Musterlösungen entgegengewirkt. Die sequenziellen Unterstützungsangebote ermöglichen durch die Aufteilung der Problemlöseaufgabe eine schrittweise eigenständige Bearbeitung der Aufgabe (FrankeBraun et al. 2008). Hinsichtlich der Selbstregulation und des Engagements von Lernenden wurden durch die Aufteilung eines Hauptziels in mehrere Teilziele bereits positive Auswirkungen beschrieben (Belland et al. 2013; Zimmerman und Kitsantas 1996).

Vor allem für leistungsschwächere Lernende sollen gestufte Lernhilfen die Möglichkeit bieten, Schritt für Schritt Teilleistungen $\mathrm{zu}$ erbringen und diese mit der entsprechenden Teillösung abzugleichen (Schmidt-Weigand et al. 2008). Weiterhin können Lernende, die die Aufgabe eigenständig bearbeiten, die Musterlösung der Lernhilfen zur Prüfung der Vollständigkeit und Richtigkeit nutzen (SchmidtWeigand et al. 2008). Zusammenfassend stellen Aufgaben mit gestuften Lernhilfen ein Format dar, das eine adaptive Unterstützung der Lernenden zu ermöglichen versucht (Franke-Braun et al. 2008; Weinert 1996). Insbesondere eignet sich der Einsatz gestufter Lernhilfen zur Strukturierung naturwissenschaftlicher Aufgaben, die Fra- 
gen zu realitätsnahen, naturwissenschaftlichen Problemen aufwerfen (Franke-Braun et al. 2008). Dabei folgen naturwissenschaftliche Aufgaben häufig einer spezifischen Abfolge von Lösungsschritten, an denen sich die Konzeption der Lösungshinweise orientieren kann (Schmidt-Weigand et al. 2008). Bezüglich der Förderung des wissenschaftlichen Denkens konnten Arnold et al. (2017) bereits die Wirksamkeit gestufter Lernhilfen bezogen auf das wissenschaftliche Denken in der gymnasialen Oberstufe zeigen, nicht aber bezogen auf methodisches Wissen und Fachwissen.

\section{Hypothesen}

Lebensweltliche, naturwissenschaftliche Problemstellungen weisen häufig einen erheblichen Komplexitätsgrad auf. Sollen Lernende im Biologieunterricht derartige Aufgabenstellungen experimentell bearbeiten, benötigen viele Schülerinnen und Schüler Unterstützung. Gestufte Lernhilfen liefern individuelle, sequenzielle Unterstützungsangebote, die vermutlich dazu führen, dass mehr Lernende in der Lage sind, die gestellten Aufgaben zu bearbeiten.

Das Vorwissen der Lernenden und die Strukturierung einer Aufgabe durch gestufte Lernhilfen beeinflussen die Anforderungen einer Aufgabe an die Lernenden und könnten daher einen Einfluss auf ihr Lernen haben. Die erste und zweite Hypothese lauten:

Das konzeptuelle bzw. prozedurale Vorwissen stellt einen Prädiktor für das konzeptuelle $\left(H l_{a}\right)$ bzw. für das prozedurale Wissen nach der Unterrichtsintervention dar $\left(H 1_{b}\right)$.

Eine Aufgabenstrukturierung durch gestufte Lernhilfen stellt einen Prädiktor für das konzeptuelle $\left(H 2_{a}\right)$ bzw. das prozedurale Wissen $\left(H 2_{b}\right)$ nach der Unterrichtsintervention dar.

Ferner wird angenommen, dass Lernende mit wenig konzeptuellem bzw. prozeduralem Vorwissen bei der Aufgabenbearbeitung mehr Unterstützung benötigen als Lernende mit einem relativ hohen konzeptuellen bzw. prozeduralen Vorwissen und daher mehr von den Lernhilfen profitieren. Die Wirksamkeit von gestuften Lernhilfen wird demnach durch konzeptuelles bzw. prozedurales Vorwissen moderiert. Die dritte Hypothese lautet:

Die Wirksamkeit der gestuften Lernhilfen auf das konzeptuelle bzw. prozedurale Wissen nach der Unterrichtsintervention ist höher je niedriger das konzeptuelle $\left(H 3_{a}\right)$ bzw. prozedurale Vorwissen $\left(H 3_{b}\right)$ ist.

\section{Methode}

\subsection{Stichprobe}

An der Studie nahmen insgesamt 187 Schülerinnen und Schüler der siebten und achten Jahrgangsstufe von acht Klassen aus Gymnasien und einer Gesamtschule 
teil. $56 \%$ der teilnehmenden Lernenden waren weiblich. Der Altersdurchschnitt lag bei 12,98 Jahren $(S D=0,82$ Jahre).

\subsection{Studiendesign}

Die Untersuchung folgte einem quasiexperimentellen Vor-Nachtest-Kontrollgruppendesign. Die Experimentalgruppe $(n=98)$ erhielt einen Arbeitsauftrag zum Experimentieren, den sie mit der Unterstützung von gestuften Lernhilfen bearbeitete, während die Kontrollgruppe $(n=89)$ lediglich den Arbeitsauftrag zum Experiment und keine gestuften Lernhilfen erhielt. Beide Gruppen unterscheiden sich demnach im Unterstützungsgrad beim Experimentieren (Abb. 1). Die Kontrollgruppe erhielt Unterstützung lediglich in Form einer direkten Instruktion vor dem Experimentieren, während die Experimentalgruppe darüber hinaus eine zum Experimentieren begleitende Unterstützung erhielt. Die Unterstützung in der Kontrollgruppe beschränkte sich auf eine Einführung in das naturwissenschaftliche Experimentieren vor dem eigenständigen Durchführen der Experimente (Unterstützung durch Erklärungen). Die Lernenden in der Experimentalgruppe erhielten zusätzlich während des eigenständigen Experimentierens Unterstützung in Form von Prompts und Erklärungen zu jedem einzelnen Schritt im Experimentierprozess (gestufte Lernhilfen). Ansonsten war der Unterricht in beiden Treatment-Bedingungen identisch.

Die Zuteilung der Klassen auf die Bedingungen Experimental- und Kontrollgruppe, d.h. welche Klasse welchem Treatment zugeteilt wurde, erfolgte per Losverfahren. Bezüglich des Geschlechts $\left(\chi^{2}(1, N=187)=0,55, p=0,55\right)$ und des Alters $(F(1,185)=0,49, p=0,49)$ sind keine signifikanten Unterschiede zwischen den Treatmentgruppen feststellbar.

Unmittelbar vor der Unterrichtseinheit und im Anschluss an diese wurde das Wissen der Lernenden mit einem identischen Wissenstest erhoben. Der Unterricht und die Befragung der Lernenden wurden von Lehramtsstudierenden eines höheren Fachsemesters durchgeführt, wobei jeder Lehramtsstudierende nach gemeinsamen Coaching zu gleichen Anteilen in beiden Treatments unterrichtete.

\subsection{Unterricht}

Im Rahmen der vierstündigen Unterrichtseinheit zum Thema Angepasstheiten von Tieren an den abiotischen Umweltfaktor Kälte wurde pro Unterrichtsstunde eine Aufgabe problemorientiert und experimentgestützt bearbeitet, die jeweils eine Überlebensstrategie von Tieren in der Kälte thematisierte.

In der ersten Stunde erhielten die Lernenden eine Einführung in das naturwissenschaftliche Arbeiten, die es ihnen ermöglichen sollte, den naturwissenschaftlichen Erkenntnisgang nachzuvollziehen. Die Lernenden beider Gruppen wurden zunächst kurz in das wissenschaftliche Arbeiten in Form von Erklärungen zu den einzelnen Schritten beim Experimentieren eingeführt.

Anschließend wurde den Lernenden auf einer Folie ein naturwissenschaftliches Phänomen in Form eines Berichts eines Forscherteams, in dem eine Beobachtung eines Phänomens dargestellt wurde, präsentiert. Die Lernenden erhielten die Aufgabe, in Kleingruppen das Phänomen im Sinne des Prozesses naturwissenschaftlicher Er- 


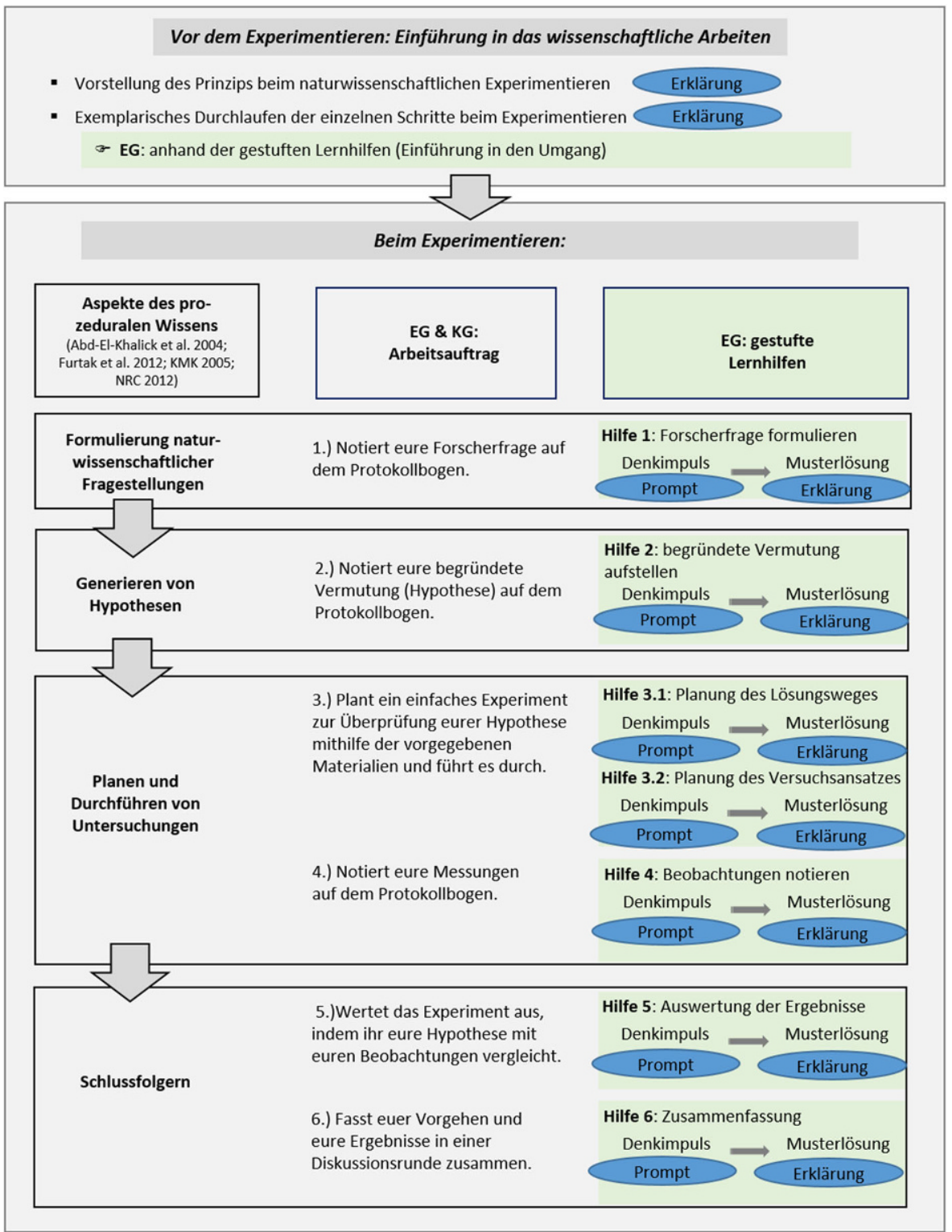

Abb. 1 Übersicht über den Ablauf des Experimentierens und die dabei zur Verfügung gestellte Unterstützung in beiden Gruppen. Elipse = Art der Unterstützung 
kenntnisgewinnung zu erklären, um den Forscherbericht zu vervollständigen. Dazu bekamen sie sechs Arbeitsaufträge, die den Problemlöseprozess in sechs Teilschritte untergliederten. Jeder Arbeitsauftrag korrespondiert mit einer der prozessbezogenen Teilfähigkeiten des naturwissenschaftlichen Erkenntnisganges (Abb. 1). Nach jedem Arbeitsauftrag wurde das Ergebnis im Plenum gesichert, so dass den Lernenden weitere Erklärungen zum Vorgehen beim Experimentieren an einem konkreten Beispiel angeboten wurden. Durch dieses Vorgehen sollten gleiche Ausgangsbedingungen bezüglich der Kenntnisse über den Ablauf und die einzelnen Teilschritte naturwissenschaftlicher Erkenntnisgewinnung für die Lernenden beider TreatmentBedingungen geschaffen werden. Die Experimentalgruppe erprobte im Rahmen dieser Einführung zusätzlich den Umgang mit den gestuften Lernhilfen, die unmittelbar den einzelnen Arbeitsaufträgen zugeordnet werden konnten (Abb. 1). So sollte gewährleistet werden, dass die Lernenden in den Experimentalklassen den Umgang mit den gestuften Lernhilfen kennenlernen, um diese in den folgenden Stunden eigenständig verwenden zu können. Im Rahmen dieser Einführungsstunde wurden die Lernenden darauf hingewiesen, die gestuften Lernhilfen auch in den folgenden Experimenten zur Unterstützung zu verwenden. Dies sollte dem häufig zu beobachtenden Phänomen des prinzipiellen Ablehnens von Hilfen vorbeugen (Kiefer und Shim 2016; Ryan et al. 2005). Insbesondere bei Schüler*innenfragen wurde auf die gestuften Lernhilfen verwiesen.

In den anschließenden beiden Unterrichtsstunden planten und führten die Lernenden eigenständig Experimente durch. Die Präsentation des Phänomens in Form eines Berichts eines Forscherteams sowie die Protokollvorlage, die eine grobe Strukturierung des Experimentierprozesses in die Teilschritte inklusive der einzelnen Arbeitsaufträge erhielt, waren strukturell identisch zur Einführungsstunde und unterschieden sich zwischen beiden Interventionsgruppen nicht.

\subsection{Konzeption der gestuften Lernhilfen}

Die gestuften Lernhilfen bestanden aus sechs inhaltlich aufeinander aufbauenden, schriftlich formulierten Karten, die durch doppeltes Falten eines DIN-A5-Blattes in einem A7-Format vorlagen. Für jeden Arbeitsauftrag konnten die Schülerinnen und Schüler auf eine Lernhilfe zurückgreifen, so dass die einzelnen Teilprozeduren des naturwissenschaftlichen Erkenntnisgewinns (Abd-El-Khalick et al. 2004; Furtak et al. 2012; KMK 2005; NRC 2012) unterstützt wurden (vgl. Abb. 1). Durch die sukzessiven Lernhilfen wurden demnach die einzelnen Teilprozeduren (prozedural), die sich inhaltlich explizit auf das zu klärende Phänomen bezogen (konzeptuell), angeleitet. Die Komplexität der Aufgabe (Klärung eines naturwissenschaftlichen Phänomens unter Verwendung naturwissenschaftlicher Arbeitsweisen) sollte durch die Nutzung der gestuften Lernhilfen schrittweise reduziert werden.

Gemäß dem eingesetzten Format enthielt jede der sechs Lernhilfen im ersten Schritt (erstes Auffalten der Karte) einen Denkimpuls, der auf die Teillösung der jeweiligen Aufgabe hinwies. Durch das erneute Auffalten der Karte konnte im zweiten Schritt die Musterlösung für die jeweilige Aufgabe eingesehen werden (Beispiel für eine Lernhilfe vgl. Abb. 2). In Anlehnung an Schmidt-Weigand et al. (2008) wies die letzte Lernhilfe zu einer Zusammenfassung der Problembearbeitung an und 


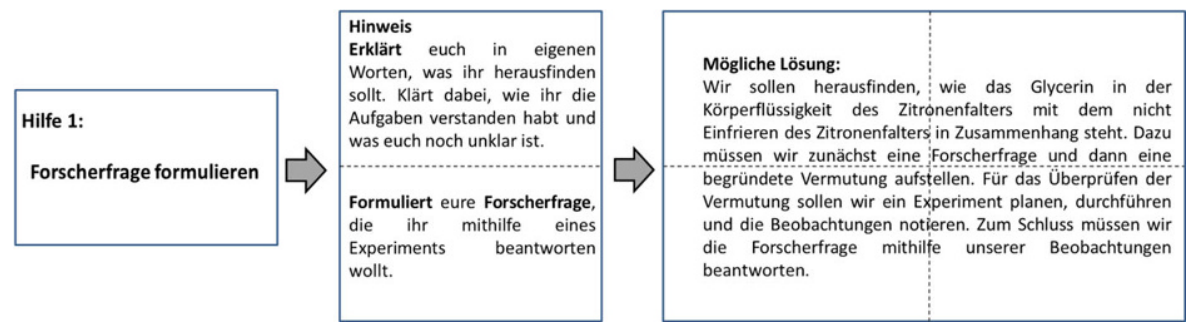

Abb. 2 Beispiel für eine Lernhilfe

forderte zur Vervollständigung des Forscherberichts auf, auf dem das Experiment basierte. Die gestuften Lernhilfen boten den Lernenden damit in zwei Schritten Unterstützung an. Zunächst in Form von Prompts als Denkimpuls, der die Lernenden dazu anregen sollte, eine bestimmte Handlung auszuführen und in einem nächsten Schritt in Form einer Erklärung, die dem Lernenden genau erläutert, was diese*r tun kann bzw. zur Überprüfung der eigenen Überlegungen dient.

Lernhilfe 1 bis 4 zielen auf die Teilprozeduren im naturwissenschaftlichen Erkenntnisgang ab, indem sie Lernenden Unterstützung bei der Formulierung der Forschungsfrage, der Generierung von Hypothesen und der Planung und Durchführung eines Experimentes anbieten. Dahingegen können Lernhilfe 5 und 6 den Aufbau von konzeptuellem Wissen unterstützen, indem sie die Lernenden anregen, aufgrund ihrer Experimente Schlussfolgerungen zu ziehen und damit allgemeine Prinzipien (z. B. im Experiment Gruppenbildung schützt vor dem Auskühlen) abzuleiten und zu reflektieren.

\subsection{Messinstrumente}

Ein selbstkonstruierter Wissenstest wurde unmittelbar vor und unmittelbar nach der Unterrichtsreihe eingesetzt. Der Wissenstest bildet das konzeptuelle Wissen zum biologischen Phänomen (vier offene Items) und das prozedurale Wissen zum Experimentieren (vier offene Items) ab.

Der Testteil zum konzeptuellen Wissen wurde in Anlehnung an die inhaltlichen Schwerpunkte des durchgeführten Unterrichts entwickelt und erfasste das Verständnis der thematisierten Inhalte. Diese Wissensfragen fokussierten dabei auf Fakten (Glaesser et al. 2009; Gott und Roberts 2008) und Prinzipien (de Jong und FergusonHessler 1996; Furtak et al. 2012) als Aspekte des konzeptuellen Wissens. Der Testteil zum prozeduralen Wissen enthielt jeweils ein offenes Item zu den Teilfähigkeiten Formulierung naturwissenschaftlicher Fragestellung, Generierung von Hypothesen, Planung von Untersuchungen und Schlussfolgern (Abd-El-Khalick et al. 2004; Furtak et al. 2012; KMK 2005; NRC 2012; Zimmerman 2005). Dabei wurde explizit von den Lernenden erwartet, aus einem beschriebenen Phänomen eine Fragestellung zu entwickeln, eine Hypothese abzuleiten, einen Experimentaufbau zu planen und auf Grundlage einer beschriebenen Beobachtung eine Schlussfolgerung zu ziehen. Der prozedurale Testteil bezog sich inhaltlich nicht auf die im Unterricht thema- 
tisierten biologischen Phänomene, sondern es sollte das im Unterricht erworbene prozedurale Wissen auf ähnliche inhaltliche Kontexte übertragen werden.

Die Schüler*innenantworten wurden mithilfe von Punkten bewertet. Die Items konnten entweder gar nicht bzw. falsch (0 Punkte), teilweise richtig (1 Punkt) oder vollständig korrekt (2 Punkte) beantwortet werden. Zur Bestimmung der Beurteilerübereinstimmung wurden jeweils $50 \%$ der Vor- und der Nachtestdaten durch eine*n zweite*n Auswerter*in unabhängig voneinander bewertet. Als Maß für die Interrater-Reliabilität wurde die Intraklassenkorrelationen bestimmt (LeBreton und Senter 2008; Wirtz und Caspar 2002). Die über alle Items gemittelte Raterübereinstimmung lag sowohl für den konzeptuellen (ICC $C_{\text {unjust, single: }}$ 0,53; Min: 0,39, Max: 0,68) und prozeduralen (ICC $C_{\text {unjust, single }}$ : 0,73; Min: 0,63, Max: 0,82) Vortest als auch für den konzeptuellen (IC $C_{\text {unjust, single }}$ : 0,68; Min: 0,58, Max: 0,77) und den prozeduralen (ICC $C_{\text {unjust, single: }}$ 0,70; Min: 0,53, Max: 0,74) Nachtest insgesamt in einem zufriedenstellenden Bereich (Wirtz und Caspar 2002).

\subsection{Statistische Auswertung}

Mittels multipler linearer Regressionen wurde analysiert, ob konzeptuelles bzw. prozedurales Vorwissen (Hypothese 1) und das Treatment (Hypothese 2), sowie die Interaktion des konzeptuellen bzw. prozeduralen Vorwissens und des Treatments (Hypothese 3) Prädiktoren des konzeptuellen bzw. prozeduralen Wissens im Nachtest darstellen. Die Regressionsmodelle wurden mit einer gewichteten Effektkodierung der kategorialen Prädiktorvariable (Treatment; Kodierung Experimentalgruppe: 1; Kodierung Kontrollgruppe: -1,17), mit Abweichungswerten vom Stichprobenmittelwert bei den metrischen Prädiktoren (konzeptuelles bzw. prozedurales Vorwissen) und mit dem Produkt der beiden Prädiktorvariablen als Interaktionsterm formuliert (Richter 2007). Als Maß für die Effektstärke der Regressionskoeffizienten wurde $\mathrm{f}^{2}$ nach folgender Formel (vgl. Cohen 1988) berechnet:

$$
f^{2}=\frac{R_{\text {Gesamt }}^{2}-R_{\mathrm{ohne}}^{2}}{1-R_{\text {Gesamt }}^{2}}
$$

mit $\mathrm{R}^{2}{ }_{\text {Gesamt }}=\mathrm{R}^{2}$ für das Gesamtmodell; $\mathrm{R}^{2}{ }_{\text {ohne }}=\mathrm{R}^{2}$ ohne Berücksichtigung des Prädiktors.

\section{Ergebnisse}

Zur Sicherstellung der Vergleichbarkeit der Treatmentgruppen bezüglich der Lernausgangslage der Lerner*innen wurde zunächst deren Vorwissen analysiert. Der Vergleich zwischen Experimental- und Kontrollgruppe mittels univariater Varianzanalyse zeigte, dass sich die Lernenden vor der Unterrichtsintervention weder im konzeptuellen $\left(F(1,185)=1,41, p=0,237, \eta_{p}{ }^{2}=0,01\right)$ noch im prozeduralen $\left(F(1,185)=2,58, p=0,110, \eta_{p}{ }^{2}=0,01\right)$ Vorwissen voneinander unterschieden (Tab. 1). 
Hypothese 1, die sich auf den Einfluss des Vorwissens auf den Wissenserwerb bezieht, Hypothese 2, die den Einfluss des Treatments auf den Wissenserwerb in den Blick nimmt, und Hypothese 3, die das Zusammenwirken von Treatment und Vorwissen thematisiert, wurden mithilfe von Regressionsanalysen untersucht. Zunächst wurden Intraklassenkorrelationen $(I C C)$ berechnet, um so mögliche Effekte der Klassenzugehörigkeit abschätzen zu können. Es zeigte sich, dass die Intraklassenkorrelationen sowohl für das konzeptuelle Vorwissen $(I C C=0,08)$ als auch für das prozedurale Vorwissen ( $\mathrm{ICC}=0,16$ ) kritische Werte größer als 0,01 aufwiesen. Dementsprechend sollte der Einfluss der Klassenzugehörigkeit im Regressionsmodell mitberücksichtigt werden, weil bereits ICCs größer als 0,01 zu einer Verzerrung der Signifikanztestung aufgrund einer Unterschätzung des Alpha-Fehlers führen (Cohen et al. 2003). Mittels des type = complex-Befehls in MPlus (Muthen und Muthen 2017) konnte die hierarchische Strukturierung der Daten aufgrund der Klassenstruktur in die Regressionsmodelle miteinbezogen werden. Mit dem Regressionsmodell zur Vorhersage des konzeptuellen Wissens im Nachtest (Tab. 2) konnten $13 \%$ der Varianz aufgeklärt werden $\left(R^{2}=0,13, S E=0,03, p<0,001 ; \chi^{2}=56,10, \mathrm{df}=3, p<0,001\right)$. Das konzeptuelle Vorwissen konnte als Prädiktor $\left(H 1_{a}\right)$ für das konzeptuelle Wissen im Nachtest bestätigt werden. Das Treatment hingegen hatte keinen signifikanten Einfluss auf das konzeptuelle Wissen im Nachtest $\left(H 2_{a}\right)$. Ebenfalls erwies sich der

Tab. 1 Mittelwerte $(M)$ mit Standardabweichungen $(S D)$ für die erreichte Punktezahl im Wissenstest (Vor- und Nachtest)

\begin{tabular}{llll}
\hline Wissen & & $\begin{array}{l}\text { Gestufte Lernhilfen } \\
(\mathrm{EG})\end{array}$ & $\begin{array}{l}\text { Ohne gestufte Lernhilfen } \\
(\mathrm{KG})\end{array}$ \\
\hline Konzeptuell & Vortest & $0,84 \pm 0,92$ & $M \pm S D$ \\
& Nachtest & $3,58 \pm 1,84$ & $1,00 \pm 0,97$ \\
\multirow{2}{*}{ Prozedural } & Vortest & $2,04 \pm 1,83$ & $3,56 \pm 1,97$ \\
& Nachtest & $3,11 \pm 2,10$ & $1,62 \pm 1,81$ \\
\hline
\end{tabular}

EG Experimentalgruppe, $K G$ Kontrollgruppe

Tab. 2 Moderierte Regression mit konzeptuellem bzw. prozeduralem Wissen im Nachtest als abhängige Variable, Treatment als unabhängige Variable und konzeptuellem bzw. prozeduralem Vorwissen als Moderator

\begin{tabular}{lllllll}
\hline Wissen & $b$ & $S E_{b}$ & $\beta$ & $p$ & $f^{2}$ \\
\hline Konzeptuelles & Konstante & 3,58 & 0,16 & 1,88 & $p<0,001$ & - \\
Wissen & Treatment & 0,06 & 0,15 & 0,04 & $p=0,66$ & 0,00 \\
& Vorwissen & 0,72 & 0,07 & 0,36 & $p<0,001$ & 0,14 \\
& Treatment*Vorwissen & $-0,01$ & 0,07 & $-0,01$ & $p=0,92$ & 0,00 \\
Prozedurales & Konstante & 2,61 & 0,19 & 1,29 & $p<0,001$ & - \\
Wissen & Treatment & 0,44 & 0,17 & 0,23 & $p=0,001$ & 0,07 \\
& & $(0,18,0,69)$ & & & & 0,17 \\
& Vorwissen & 0,42 & 0,07 & 0,37 & $p<0,001$ & 0,00 \\
& & $(0,27,0,57)$ & & & & \\
& Treatment*Vorwissen & $-0,06$ & 0,06 & $-0,05$ & $p=0,36$ & 0 \\
& $(-0,20,0,07)$ & & & & & \\
\hline
\end{tabular}

$R_{\text {konzeptuell }}^{2}=0,13(p<0,001)$ und $R_{\text {prozedural }}^{2}=0,22(p<0,01)$ 
Interaktionsterm (Treatment*konzeptuelles Vorwissen) als nicht signifikant $\left(H 3_{a}\right)$. Das konzeptuelle Vorwissen stellt somit keinen Moderator für die Wirksamkeit der gestuften Lernhilfen dar. Bezogen auf das prozedurale Wissen (Tab. 2) konnte das lineare multiple Regressionsmodell mit den beiden Prädiktoren (prozedurales Vorwissen, Treatment) und dem Interaktionsterm rund ein Fünftel der Varianz für das prozedurale Wissen im Nachtest erklären $\left(R^{2}=0,21, \mathrm{SE}=0,07 ; p<0,01 ; \chi^{2}=529,57\right.$, $\mathrm{df}=3 ; p<0,001)$. Das prozedurale Vorwissen $\left(H 1_{b}\right)$ sowie das Treatment $\left(H 2_{b}\right)$ erwiesen sich als signifikante Prädiktoren für das prozedurale Wissen im Nachtest. Lernende, die mit gestuften Lernhilfen arbeiteten, erzielten höhere Leistungen im prozeduralen Nachtest als Lernende, die keine gestuften Lernhilfen verwendeten. Der Interaktionsterm (Treatment*prozedurales Vorwissen) war nicht signifikant $\left(H 3_{b}\right)$. Das prozedurale Vorwissen stellt demnach keinen Moderator für die Wirksamkeit der gestuften Lernhilfen dar. Schülerinnen und Schüler profitieren also unabhängig von ihrem Vorwissen in gleichem Ausmaß von den gestuften Lernhilfen.

Um die nichtsignifikanten Prädiktoren abzusichern, wurde eine Sensitivitätsanalyse $(\alpha=0,05,1-\beta=0,90)$ mit $G^{*}$ Power (Faul et al. 2009) durchgeführt. Diese zeigt, dass Effekte mit einer Effektstärke von $\mathrm{f}^{2}>0,05$ nachweisbar wären. Demnach sind mit dem Studiendesign selbst kleine Effekte nachweisbar und eine zu geringe Testpower ist als potenzielle Erklärung für die Nulleffekte unwahrscheinlich.

\section{Diskussion}

Zentrales Anliegen dieser Studie war es, den Einfluss gestufter Lernhilfen auf den konzeptuellen und prozeduralen Lernerfolg beim Experimentieren im Biologieunterricht und deren Wechselwirkung mit dem Vorwissen der Lernenden zu untersuchen.

\subsection{Konzeptuelles Wissen}

Die Analysen des konzeptuellen Wissens zeigen, dass nicht die gestuften Lernhilfen (Treatment), sondern nur das konzeptuelle Vorwissen einen Prädiktor für das konzeptuelle Wissen im Nachtest darstellt. Die Hypothese $H 1_{a}$ wird somit gestützt, während die Hypothese $H 2_{a}$ nicht gestützt werden kann. Weiterhin zeigen die Befunde, dass Vorwissen kein Moderator für die Wirksamkeit der gestuften Lernhilfen ist. Demnach wird Hypothese $H 3_{a}$ nicht gestützt. Offenbar hatten die gestuften Lernhilfen keinen Einfluss auf das konzeptuelle Wissen. Eine mögliche Erklärung liegt in der Gestaltung der gestuften Lernhilfen. Diese zielte auf eine Unterstützung der prozessbezogenen Teilfähigkeiten des Experimentierens im Rahmen des naturwissenschaftlichen Erkenntnisweges ab. Der Aufbau konzeptuellen Wissens könnte daher nur implizit durch die gestuften Lernhilfen unterstützt worden sein, indem sich die prozessbezogenen Teilfähigkeiten auf die Konzepte der Überwinterungsstrategien bezogen. Aufgrund der Komplexitätsreduktion beim Experimentierprozess durch die gestuften Lernhilfen wurde ein indirekter positiver Effekt bezüglich des Durchdringens der Inhalte erwartet. Obwohl in einigen Studien nachgewiesen werden konnte, dass aus prozeduralem Wissen, konzeptuelles Wissen hervorgehen kann (Anderson et al. 2001), hat sich dieser Befund in der vorliegenden Studie wie 
auch in anderen Studien (vgl. zusammengefasst bei Rittle-Johnson et al. 2015) nicht gezeigt. Generell scheinen sich verschiedene unterstützende Maßnahmen beim Experimentieren unterschiedlicher Spezifität in ihrer Effektivität auf das konzeptuelle Lernen nicht zu unterscheiden (Lazonder und Harmsen 2016). Insgesamt erscheinen allerdings unterstützende Maßnahmen beim Experimentieren eher weniger effektiv für das konzeptionelle Lernen zu sein, weil die angebotene Unterstützung explizit auf Unterstützung der Lernenden beim Experimentieren ausgerichtet ist (Lazonder und Harmsen 2016).

\subsection{Prozedurales Wissen}

Beim prozeduralen Wissen erwiesen sich das prozedurale Vorwissen sowie die gestuften Lernhilfen als Prädiktoren für das prozedurale Vorwissen im Nachtest. Hypothese $H 1_{b}$ und $H 2_{b}$ werden somit gestützt.

Dieser Befund unterstützt die Annahme, dass Lernende von strukturierenden Maßnahmen profitieren (Kirschner et al. 2006; Mayer 2004; Oksa et al. 2010). Die Wirksamkeit gestufter Lernhilfen kann demnach als unterstützende Maßnahme (Franke-Braun et al. 2008) zur Förderung des prozeduralen Wissens beim Experimentieren in der Mittelstufe angenommen werden. Ein ähnlicher Befund wurde bereits für die Wirksamkeit gestufter Lernhilfen zur Förderung des prozeduralen Wissens in der gymnasialen Oberstufe nachgewiesen (Arnold et al. 2017).

Die Annahme, dass die Wirksamkeit der gestuften Lernhilfen vom prozeduralen Vorwissen abhängt, konnte im Rahmen der Studie nicht bestätigt werden. Hypothese $\mathrm{H} 3_{\mathrm{b}}$ wird nicht gestützt. Somit kann die Annahme, dass unterstützende Maßnahmen Lernende mit geringem Vorwissen besser unterstützen als Lernende mit hohem Vorwissen (Kalyuga 2013), in dieser Studie nicht bestätigt werden. Der Einsatz gestufter Lernhilfen ist demnach unabhängig vom Vorwissen für alle Lernenden wirksam.

\subsection{Limitationen}

Die Schülerinnen und Schüler wurden zwar im Rahmen der Studie darauf hingewiesen, die gestuften Lernhilfen zur Unterstützung während der Aufgabenbearbeitung zu nutzen, dennoch kann nicht gewährleistet werden, dass diese von allen Lernenden adäquat verwendet wurden. Es ist nicht auszuschließen, dass einige Lernenden, insbesondere Lernende mit niedrigerem Vorwissen, die Lernhilfen nicht verwendet haben. In Studien hat sich gezeigt, dass leistungsschwächere Lerner eher dazu neigen, angebotene Unterstützung nicht anzunehmen bzw. sich keine Hilfe zu suchen (Ryan et al. 2005; Ryan und Shin 2011). Des Weiteren kann mit dem Studiendesign ohne eine Erfassung der tatsächlichen Nutzung der Lernhilfen und ohne Vergleich mit einer Musterlösungen-Kontrollgruppe die Frage nicht abschließend geklärt werden, ob tatsächlich die Stufung der Lernhilfen relevant ist oder vielleicht das Anbieten der Musterlösung im letzten Schritt der Lernhilfe den entscheidenden Aspekt darstellt. In Folgestudien sollte deshalb der tatsächliche Gebrauch der gestuften Lernhilfen erhoben werden, um durch diese Implementationskontrolle die Befunde abzusichern. 
Es ist weiterhin nicht auszuschließen, dass die Lernenden aufgrund des für sie neuen Aufgabenformats, das Potenzial der gestuften Lernhilfen nicht vollständig nutzen konnten. Wahser und Sumfleth (2008) zeigten, dass Lernende von einem Training zum Umgang mit eingesetzten Strukturierungshilfen profitieren. Unter Berücksichtigung der verhältnismäßig kurzen Intervention ist nicht auszuschließen, dass Lernende von einem intensiveren Methodentraining zur Arbeit mit gestuften Lernhilfen im experimentellen Biologieunterricht profitiert hätten. Die Implementation eines Methodentrainings über eine längere Unterrichtssequenz ist für Folgeuntersuchungen zu erwägen. Um sichere Aussagen über die Wirksamkeit der gestuften Lernhilfen treffen zu können, wäre darüber hinaus eine Analyse zur Nutzung der einzelnen gestuften Lernhilfen relevant.

Die Stichprobe bestand vorwiegend aus Lernenden von Gymnasien. In Gesamtschulklassen könnte unter Umständen mit einer höheren Heterogenität, gerade auch im Bereich des Vorwissens, gerechnet werden. Es ist demnach nicht auszuschließen, dass die Befunde dieser Studie nicht auf alle Schultypen generalisiert werden können. Eine Ausweitung der Stichprobe auf Gesamtschulen ist anzustreben.

In dieser Studie wurde zwar die Klassenzugehörigkeit als möglicher beeinflussender Faktor in die Analyse einbezogen, allerdings experimentierten die Schüler*innen in Kleingruppen zusammen, was potenziell ebenfalls die Befunde in dieser Studie beeinflusst haben könnte. In weiteren Untersuchungen sollte auch diese Ebene berücksichtigt werden.

\subsection{Implikationen für den Biologieunterricht und die weitere Forschung}

Zusammenfassend waren die gestuften Lernhilfen im experimentellen Biologieunterricht hinsichtlich der Förderung prozeduralen Wissens erfolgreich. Die Ergebnisse geben Hinweise auf die Wirksamkeit eines Unterstützungsformats beim Experimentieren im Biologieunterricht, das eine Aufgabenbearbeitung zwischen expliziter Anleitung und offenem Problemlösen bietet (Hänze et al. 2007). Insgesamt zeigte sich, dass eine Kombination aus einer Einführung in die wissenschaftliche Arbeitsweise in Form von Erklärungen vor dem eigenständigen Experimentieren und eine gezielte, spezifische Unterstützung während des Experimentierprozesses durch die gestuften Lernhilfen für Lernende effektiver zu sein scheint, als nur eine Einführung in die wissenschaftliche Arbeitsweise losgelöst vom eigenständigen Experimentieren. Auch in der Metaanalyse von Lazonder und Harmsen (2016) lassen sich Hinweise darauf finden, dass Experimentieren mit spezifischeren Arten der Unterstützung für Lernende effektiver ist.

Vor dem Hintergrund der Forderung, didaktische Maßnahmen zur Differenzierung und Individualisierung bereitzustellen (Helmke 2015), wurde das Format der gestuften Lernhilfen vorgeschlagen (Hänze et al. 2007). Die Ergebnisse dieser Studie geben Hinweise auf die Wirksamkeit gestufter Lernhilfen bei der Förderung prozessbezogener Teilfähigkeiten zum Experimentieren. Gestufte Lernhilfen könnten demnach für das selbstständige experimentelle Problemlösen zur Verfügung gestellt werden, um den Prozess des Experimentierens bei der gezielten Erarbeitung bestimmter prozessbezogener Teilfähigkeiten zu unterstützen. Allerdings deuten die Befunde in dieser Studie darauf hin, dass Lernende unterschiedlicher Vorwissens- 
stände von gestuften Lernhilfen nicht in unterschiedlichem Maße profitieren. So liefert diese Untersuchung keinen Hinweis darauf, dass Lernende mit wenig Vorwissen besonders von gestuften Lernhilfen profitieren. Sie stellen vielmehr eine Maßnahme zur Unterstützung aller Lernenden unabhängig des Vorwissens dar.

Lernende, denen die Nutzung von Hilfestellungen freigestellt ist, haben häufig Schwierigkeiten damit ihren eigenen Lernprozess zu überwachen und dementsprechend angemessene Hilfen zu nutzen (Clarebout und Elen 2006; Ryan und Shin 2011). In computerbasierten Lernsettings zeigte sich, dass Lernende dazu neigen, Hilfen nicht adäquat zu nutzen und z. B. Hinweise überspringen und direkt zu den Lösungen gehen (Aleven et al. 2016; Bartholomé et al. 2006). Darüber hinaus zeigen sich Unterschiede im Hilfesuchverhalten in Abhängigkeit vom Leistungsniveau der Lernenden (Ryan et al. 2005; Ryan und Shin 2011). Leistungsstärkere Lernende erkennen das Hilfesuchen- und annehmen eher als Lernstrategie (Ryan et al. 2005) und können dieses auch angemessen anwenden (Bartholomé et al. 2006), während leistungsschwächere Lernende in diesem Punkt weniger effektiv sind (Bartholomé et al. 2006) bzw. es eher vermeiden, sich Hilfe zu suchen, um ihre Defizite nicht offenzulegen und sich vor den anderen nicht bloßzustellen (Ryan et al. 2005). Um das Potenzial der Lernhilfen ausschöpfen zu können, erscheint es gerade deswegen günstiger, die Anforderungen an Lernende zunächst zu reduzieren, insbesondere, wenn neuer Lernstoff eingeführt wird bzw. neue Kompetenzen erworben werden sollen. Deswegen wurden in dieser Studie die Lernenden nicht vor die Wahl gestellt, ob sie die Hilfen nutzen. Dennoch sollte gerade mit dem Blick auf den adaptiven Charakter von gestuften Lernhilfen (Franke-Braun et al. 2008; Weinert 1996) sowie der eigentlichen Intention beim Einsatz von gestuften Lernhilfen, die Entscheidung über die Nutzung in die Hand der Lernenden zu legen (Leisen 2013), diese Verbindlichkeit der Nutzung in einer längeren Unterrichtssequenz zurückgefahren werden. Wenn Lernende gelernt haben, adäquat einzuschätzen, wann sie Hilfen benötigen, kann ihnen so ein noch selbstbestimmteres Experimentieren ermöglicht werden. Das Potenzial des adaptiven Ansatzes für den inklusiven Biologieunterricht, z.B. die Eignung als unterstützende Maßnahme speziell für Lernende mit wenig Vorwissen, sollte in weiterführenden Studien fokussiert werden. Diesbezüglich wird eine Ausweitung der Stichprobe auf weitere Schulformen angestrebt. Außerdem soll die Erhebung weiterer relevanter Variablen (z.B. Gruppenzugehörigkeit, Auswertung der Protokolle der Lernenden, motivationale Variablen) berücksichtigt werden. Weiterhin soll der Einfluss einer längeren Trainingsphase für den Umgang mit gestuften Lernhilfen sowie die Relevanz der freiwilligen Nutzung der gestuften Hilfen sowie günstige Rahmenbedingungen für die Nutzung dieser für Schüler*innen mit weniger Vorwissen untersucht werden.

Funding Open Access funding enabled and organized by Projekt DEAL.

Open Access Dieser Artikel wird unter der Creative Commons Namensnennung 4.0 International Lizenz veröffentlicht, welche die Nutzung, Vervielfältigung, Bearbeitung, Verbreitung und Wiedergabe in jeglichem Medium und Format erlaubt, sofern Sie den/die ursprünglichen Autor(en) und die Quelle ordnungsgemäß nennen, einen Link zur Creative Commons Lizenz beifügen und angeben, ob Änderungen vorgenommen wurden. 
Die in diesem Artikel enthaltenen Bilder und sonstiges Drittmaterial unterliegen ebenfalls der genannten Creative Commons Lizenz, sofern sich aus der Abbildungslegende nichts anderes ergibt. Sofern das betreffende Material nicht unter der genannten Creative Commons Lizenz steht und die betreffende Handlung nicht nach gesetzlichen Vorschriften erlaubt ist, ist für die oben aufgeführten Weiterverwendungen des Materials die Einwilligung des jeweiligen Rechteinhabers einzuholen.

Weitere Details zur Lizenz entnehmen Sie bitte der Lizenzinformation auf http://creativecommons.org/ licenses/by/4.0/deed.de.

\section{Literatur}

Abd-El-Khalick, F., Boujaoude, S., Duschl, R., Lederman, N. G., Mamlok-Naaman, R., Hofstein, A., Niaz, M., Treagust, D., \& Tuan, H. (2004). Inquiry in science education: International perspectives. Science Education, 88, 397-419. https://doi.org/10.1002/sce.10118.

Abels, S. (2015). Scaffolding inquiry-based science and chemistry education in inclusive classroom. In N. L. Yates (Hrsg.), New developments in science education research (S. 77-95). New York: Nova Science.

Aleven, V., Roll, I., McLaren, B. M., \& Koedinger, K. R. (2016). Help helps, but only so much: Research on help seeking with. Intelligent Tutoring Systems. International Journal of Artificial Intelligence in Education, 26, 205-223. https://doi.org/10.1007/s40593-015-0089-1.

Anderson, L. W., Krathwohl, D. R., Airasian, P. W., Cruikshank, K. A., Mayer, R. E., Pintrich, P. R., Raths, J., \& Wittrock, M.C. (2001). A taxonomy for learning, teaching, and assessing: A revision of bloom's taxonomy of educational objectives. New York: Longman.

Arnold, J., Kremer, K., \& Mayer, J. (2014). Understanding students' experiments-What kind of support do they need in inquiry tasks? International Journal of Science Education, 36(15-16), 2719-2749. https://doi.org/10.1080/09500693.2014.930209.

Arnold, J., Kremer, K., \& Mayer, J. (2017). Scaffolding beim Forschenden Lernen. Eine empirische Untersuchung zur Wirksamkeit von Lernunterstützungen. Zeitschrift für Didaktik der Naturwissenschaften, 23, 21-37. https://doi.org/10.1007/s40573-016-0053-0.

Bartholomé, T., Stahl, E., Pieschl, S., \& Bromme, R. (2006). What matters in help-seeking? A study of help effectiveness and learner-related factors. Computers in Human Behavior, 22(1), 113-129. https://doi. org/10.1016/j.chb.2005.01.007.

Bell, R. L., Blair, L.M., Crawford, B. A., \& Lederman, N. G. (2003). Just do it? Impact of a science apprenticeship program on high school students' understandings of the nature of science and scientific inquiry. Journal of research in science teaching, 40(5), 487-509. https://doi.org/10.1002/tea.10086.

Belland, B.R., Kim, C., \& Hannafin, M. J. (2013). A framework for designing scaffolds that improve motivation and cognition. Educational Psychologist, 48(4), 243-270. https://doi.org/10.1080/00461520. 2013.838920 .

Blanchard, M., Southerland, S. H., Osborne, J. W., Sampson, V. D., Annetta, L. A., \& Granger, E. M. (2010). Is inquiry possible in light of accountability? A quantitative comparison of the relative effectiveness of guided inquiry and verification laboratory instruction. Science Education, 94(4), 577-616. https:// doi.org/10.1002/sce.20390.

Clarebout, G., \& Elen, J. (2006). Tool use in computer-based learning environments: towards a research framework. Computers in Human Behavior, 22(3), 389-411. https://doi.org/10.1016/j.chb.2004.09. 007.

Cohen, J. (1988). Statistical power analysis for the behavioral sciences (2. Aufl.). Hillsdale: Erlbaum.

Cohen, J., Cohen, P., West, S., \& Aiken, L. S. (2003). Applied multiple regression/correlation analysis for the behavioral sciences (3. Aufl.). Mahwah: Erlbaum.

Colburn, A. (2000). An inquiry primer. Science Scope, 23(6), 42-44.

Dohn, N. B. (2013). Situational interest in engineering design activities. International Journal of Science Education, 35(12), 37-41. https://doi.org/10.1080/09500693.2012.757670.

Einsiedler, W., \& Hardy, I. (2010). Kognitive Strukturierung im Unterricht: Einführung und Begriffsklärung. Unterrichtswissenschaft, 38(3), 194-209.

Faul, F., Erdfelder, E., Buchner, A., \& Lang, A. G. (2009). Statistical power analyses using G* power 3.1: tests for correlation and regression analyses. Behavior research methods, 41(4), 1149-1160.

Flick, L., \& Lederman, N. (2006). Introduction. In L. Flick \& N. Lederman (Hrsg.), Scientific inquiry and nature of science (S. ix-xviii). Dordrecht: Springer. 
Franke-Braun, G., Schmidt-Weigand, F., Stäudel, L., \& Wodzinski, R. (2008). Aufgaben mit gestuften Lernhilfen - ein besonderes Aufgabenformat zur kognitiven Aktivierung der Schülerinnen und Schüler und zur Intensivierung der sachbezogenen Kommunikation. In Kasseler Forschungsgruppe (Hrsg.), Lernumgebungen auf dem Prüfstand: Zwischenergebnisse aus den Forschungsprojekten (S. 27-42). Kassel: Kassel University Press.

Furtak, E. M., Seidel, T., Iverson, H., \& Briggs, D. C. (2012). Experimental and quasi-experimental studies of inquiry-based science teaching: a meta-analysis. Review of Educational Research, 82(3), 300-329. https://doi.org/10.3102/0034654312457206.

Glaesser, J., Gott, R., Roberts, R., \& Cooper, B. (2009). Underlying success in open-ended investigations in science: Using qualitative comparative analysis to identify necessary and sufficient conditions. Research in Science \& Technological Education, 27(1), 5-30. https://doi.org/10.1080/ 02635140802658784.

Gott, R., \& Roberts, R. (2008). Concepts of evidence and their role in open-ended practical investigations and scientific literacy; background to published papers. Durham: Durham University.

Hammann, M., \& Prenzel, M. (2008). Ergebnisse des internationalen PISA Naturwissenschaftstests 2006. Der mathematische und naturwissenschaftliche Unterricht, 61(2), 67-74.

Hammann, M., Phan, T.T.H., \& Bayrhuber, H. (2007). Experimentieren als Problemlösen: Lässt sich das SDDS-Modell nutzen, um unterschiedliche Dimensionen beim Experimentieren zu messen? In M. Prenzel, I. Gogolin \& H.-H. Krüger (Hrsg.), Kompetenzdiagnostik (Zeitschrift für Erziehungswissenschaft: Sonderheft 8, S. 33-49). Wiesbaden: Springer VS. https://doi.org/10.1007/978-3-53190865-6_3.

Hänze, M., Schmidt-Weigand, F., \& Blum, S. (2007). Mit gestuften Lernhilfen selbständig lernen und arbeiten. In K. Rabenstein \& S. Reh (Hrsg.), Kooperatives und selbständiges Lernen von Schülern (S. 197-208). Wiesbaden: VS. https://doi.org/10.1007/978-3-531-90418-4_10.

Hänze, M., Schmidt-Weigand, F., \& Stäudel, L. (2010). Gestufte Lernhilfen. In S. Boller \& R. Lau (Hrsg.), Individuelle Förderung durch Innere Differenzierung. Ein Praxishandbuch für Lehrerinnen und Lehrer der Sekundarstufe II (S. 63-73). Weinheim: Beltz.

Hardy, I. (2012). Kognitive Strukturierung - Empirische Zugänge zu einem heterogenen Konstrukt der Unterrichtsforschung. In F. Hellmich, S. Förster \& F. Hoya (Hrsg.), Bedingungen des Lehrens und Lernens in der Grundschule. Bilanz und Perspektiven (Bd. 16, S. 51-62). Wiesbaden: VS. https://doi. org/10.1007/978-3-531-19137-9_4.

Hardy, I., Jonen, A., Möller, K., \& Stern, E. (2006). Effects of instructional support within constructivist environments for elementary school students' understanding of "floating and sinking". Journal of Educational Psychology, 98, 307-326. https://doi.org/10.1037/0022-0663.98.2.307.

Helmke, A. (2015). Unterrichtsqualität und Lehrerprofessionalität. Diagnose, Evaluation und Verbesserung des Unterrichts (6. Aufl.). Seelze: Klett-Kallmeyer.

Hmelo-Silver, C. E., Duncan, R. G., \& Chinn, C. A. (2007). Scaffolding and achievement in problem-based and inquiry learning: A response to Kirschner, Sweller, and Clark (2006). Educational Psychologist, 42(2), 99-107. https://doi.org/10.1080/00461520701263368.

Hodson, D. (1998). Is this really what scientists do? Seeking a more authentic science in and beyond the school laboratory. In L. Wellington (Hrsg.), Practical work in school science: Which way now? (S. 93-107). London: Routledge.

Hofstein, A., \& Lunetta, V.N. (2004). The laboratory in science education: Foundations for the twentyfirst century. International Journal of Science Education, 88(1), 28-54. https://doi.org/10.1002/sce. 10106.

de Jong, T. (2005). The guided discovery principle in multimedia learning. In R.E. Mayer (Hrsg.), The Cambridge handbook of multimedia learning (S. 215-228). Cambridge: Cambridge University Press. https://doi.org/10.1017/CBO9780511816819.015.

de Jong, T. (2019). Moving towards engaged learning in STEM domains; there is no simple answer, but clearly a road ahead. Journal of Computer Assisted Learning, 35(2), 153-167. https://doi.org/10. 1111/jcal.12337.

de Jong, T., \& Ferguson-Hessler, M. G. M. (1996). Types and qualities of knowledge. Educational Psychologist, 31(2), 105-113. https://doi.org/10.1207/s15326985ep3102_2.

de Jong, T., \& van Joolingen, W.R. (1998). Scientific discovery learning with computer simulations of conceptual domains. Review of Educational Research, 68(2), 179-201. https://doi.org/10.3102/ 00346543068002179 .

de Jong, T., \& Lazonder, A.W. (2014). The guided discovery learning principles in multimedia learning. In R.E. Mayer (Hrsg.), Cambridge handbooks in psychology: The Cambridge handbook of multime- 
dia learning (2. Aufl., S. 371-390). New York: Cambridge University Press. https://doi.org/10.1017/ CBO9781139547369.019.

Kalyuga, S. (2007). Expertise reversal effect and its implications for learner-tailored instruction. Educational Psychology Review, 19, 509-539. https://doi.org/10.1007/s10648-007-9054-3.

Kalyuga, S. (2013). Effects of learner prior knowledge and working memory limitations on multimedia learning. Procedia-Social and Behavioral Sciences, 83, 25-29. https://doi.org/10.1016/j.sbspro. 2013.06.005.

Kalyuga, S., Ayres, P., Chandler, P., \& Sweller, J. (2003). The expertise reversal effect. Educational Psychologist, 38(1), 23-31. https://doi.org/10.1207/S15326985EP3801_4.

Kalyuga, S., Chandler, P., \& Sweller, J. (2001). Learner experience and efficiency of instructional guidance. Educational Psychology, 21(1), 5-23. https://doi.org/10.1080/01443410124681.

Kiefer, S. M., \& Shim, S.S. (2016). Academic help seeking from peers during adolescence: The role of social goals. Journal of Applied Developmental Psychology, 42, 80-88. https://doi.org/10.1016/j. appdev.2015.12.002.

Kirschner, P., Sweller, J., \& Clark, R. E. (2006). Why minimal guidance during instruction does not work: An analysis of the failure of constructivist, discovery, problem-based, experiential, and inquiry-based teaching. Educational Psychologist, 41(2), 75-86. https://doi.org/10.1207/s15326985ep4102_1.

Klahr, D. (2000). Exploring science: The cognition and development of discovery processes. Cambridge: MIT Press.

Kleickmann, T., Vehmeyer, V., \& Möller, K. (2010). Zusammenhänge zwischen Lehrervorstellungen und kognitivem Strukturieren im Unterricht am Beispiel von Scaffolding-Maßnahmen. Unterrichtswissenschaft, 38(3), 210-228.

KMK (Sekretariat der Ständigen Konferenz der Kultusminister der Länder in der Bundesrepublik Deutschland) (2005). Beschlüsse der Kultusministerkonferenz. Bildungsstandards im Fach Biologie für den Mittleren Schulabschluss. Beschluss vom 16.12.2004. München: Luchterhand.

Kollöffel, B., \& de Jong, T. (2013). Conceptual understanding of electrical circuits in secondary vocational engineering education: Combining traditional instruction with inquiry learning in a virtual lab. Journal of Engineering Education, 102(3), 375-393. https://doi.org/10.1002/jee.20022.

Krajcik, J., Blumenfeld, P.C., Marx, R.W., Bass, K.M., Fredricks, J., \& Soloway, E. (1998). Inquiry in project-based science classrooms: Initial attempts by middle school students. Journal of the Learning Sciences, 7(3-4), 313-350. https://doi.org/10.1080/10508406.1998.9672057.

Lazonder, A. W., \& Harmsen, R. (2016). Meta-analysis of inquiry-based learning. Review of Educational Research, 86(3), 681-718. https://doi.org/10.3102/0034654315627366.

LeBreton, J.M., \& Senter, J.L. (2008). Answers to 20 questions about Interrater reliability and Interrater agreement. Organizational Research Methods, 11(4), 815-852. https://doi.org/10.1177/ 1094428106296642.

Leisen, J. (1999). Methodenhandbuch deutschsprachiger Fachunterricht DFU. Bonn: Varus.

Leisen, J. (2013). Handbuch Sprachförderung im Fach (Sprachsensibler Fachunterricht in der Praxis Grundlagenwissen, Anregungen und Beispiele für die Unterstützung von sprachschwachen Lernern und Lernern mit Zuwanderungsgeschichte beim Sprechen, Lesen, Schreiben und Üben im Fach). Stuttgart: Klett.

Mayer, R.E. (2004). Should there be a three-strikes rule against pure discovery learning? The case for guided methods of instruction. American Psychologist, 59(1), 14-19. https://doi.org/10.1037/0003066X.59.1.14.

MSW NRW (Ministerium für Schule und Weiterbildung des Landes NRW) (2013). Kernlehrplan für die Gesamtschule - Sekundarstufe I in Nordrhein-Westfalen. Naturwissenschaften. Biologie, Chemie, Physik (2. Aufl., Bd. 2013). Frechen: Ritterbach.

Muthen, L. K., \& Muthen, B. O. (2017). MPlus user guide (8. Aufl.). Los Angeles: Muthen \& Muthen.

NRC (National Research Council) (2012). A framework for K-12 science education: Practices, crosscutting concepts, and core ideas. Washington: National Academy Press.

OECD (Organisation for Economic Cooperation and Development) (2007). PISA 2006. Science competencies for tomorrow's world. Paris: OEC.

Oksa, A., Kalyuga, S., \& Chandler, P. (2010). Expertise reversal effect in using explanatory notes for readers of Shakespearean text. Instructional Science, 38(3), 217-236. https://doi.org/10.1007/s11251009-9109-6.

Osborne, J., Collins, S., Ratcliffe, M., Millar, R., \& Duschl, R. (2003). What "ideas-about-science" should be taught in school science? A Delphi study of expert community. Journal of Research in Science Teaching, 40(7), 692-720. https://doi.org/10.1002/tea.10105. 
Paas, F., Renkl, A., \& Sweller, J. (2003). Cognitive load theory and instructional design: Recent developments. Educational Psychologist, 38(1), 1-4. https://doi.org/10.1207/S15326985EP3801_1.

Papadouris, N., \& Constantinou, C.P. (2009). A methodology for integrating computer-based learning tools in science curricula. Journal of Curriculum Studies, 41(4), 521-538. https://doi.org/10.1080/ 00220270802123946.

Richter, T. (2007). Wie analysiert man Interaktionen von metrischen und kategorialen Prädiktoren? Nicht mit Median-Splits! Zeitschrift für Medienpsychologie, 19(3), 116-125. https://doi.org/10.1026/16176383.19.3.116.

Rittle-Johnson, B., Schneider, M., \& Star, J.R. (2015). Not a one-way street: Bidirectional relations between procedural and conceptual knowledge of mathematics. Educational Psychology Review, 27, 587-597. https://doi.org/10.1007/s10648-015-9302-x.

Ryan, A. M., \& Shin, H. (2011). Help-seeking tendencies during early adolescence: An examination of motivational correlates and consequences for achievement. Learning and Instruction, 21(2), 247-256. https://doi.org/10.1016/j.learninstruc.2010.07.003.

Ryan, A. M., Patrick, H., \& Shim, S. O. (2005). Differential profiles of students identified by their teacher as having avoidant, appropriate, or dependent help-seeking tendencies in the classroom. Journal of Educational Psychology, 97(2), 275-285. https://doi.org/10.1037/0022-0663.97.2.275.

Sadeh, I., \& Zion, M. (2012). Which type of inquiry project do high school biology students prefer: open or guided? Research in Science Education, 42(5), 831-848. https://doi.org/10.1007/s11165-011-92229.

Schiepe-Tiska, A., Rönnebeck, S., Schöps, K., Neumann, K., Schmidtner, S., Parchmann, I., \& Prenzel, M. (2016). Naturwissenschaftliche Kompetenz in PISA 2015 - Ergebnisse des internationalen Vergleichs mit einem modifizierten Testansatz. In K. Reiss, C. Sälzer, A. Schiepe-Tiska, E. Klieme \& O. Köller (Hrsg.), PISA 2015. Eine Studie zwischen Kontinuität und Innovation (S. 45-98). Münster: Waxmann.

Schmidt-Weigand, F., Franke-Braun, G., \& Hänze, M. (2008). Erhöhen gestufte Lernhilfen die Effektivität von Lösungsbespielen? Unterrichtswissenschaft, 36(4), 365-384.

Schmidt-Weigand, F., Hänze, M., \& Wodzinski, R. (2009). Complex problem solving and worked examples. The role of prompting strategic behavior and fading-in solution steps. Zeitschrift für Pädagogische Psychologie, 23(2), 129-138. https://doi.org/10.1024/1010-0652.23.2.129.

Stäudel, L., Franke-Braun, G., \& Schmidt-Weigand, F. (2007). Komplexität erhalten-auch in heterogenen Lerngruppen: Aufgaben mit gestuften Lernhilfen. CHEMKON: Forum für Unterricht und Didaktik, 14(3), 115-122.

Sweller, J., van Merrienboer, J.J.G., \& Paas, F. G. W.C. (1998). Cognitive architecture and instructional design. Educational Psychology Review, 10(3), 251-296. https://doi.org/10.1023/A:1022193728205.

Wahser, I., \& Sumfleth, E. (2008). Training experimenteller Arbeitsweisen zur Unterstützung kooperativer Kleingruppenarbeit im Fach Chemie. Zeitschrift für Didaktik der Naturwissenschaften, 14, 219-241.

Weinert, F. (1996). Psychologie des Lernens und der Instruktion. Göttingen: Hogrefe.

Wirth, J., Thillmann, H., Künsting, J., Fischer, H.E., \& Leutner, D. (2008). Das Schülerexperiment im naturwissenschaftlichen Unterricht. Bedingungen der Lernförderlichkeit einer verbreiteten Lehrmethode aus instruktionspsychologischer Sicht. Zeitschrift für Pädagogik, 54(3), 361-375.

Wirtz, M. A., \& Caspar, F. (2002). Beurteilerübereinstimmung und Beurteilerreliabilität. Methoden zur Bestimmung und Verbesserung der Zuverlässigkeit von Einschätzungen mittels Kategoriensystemen und Ratingskalen. Göttingen: Hogrefe.

Zimmerman, B. J. (2005). The development of scientific reasoning skills: What psychologists contribute to an understanding of elementary science learning. Report to the national research council, committee on science learning kindergarten through eighth grade. Illinois: Illinois State University.

Zimmerman, B.J., \& Kitsantas, A. (1996). Self-regulated learning of a motoric skill: The role of goal setting and self-monitoring. Journal of Applied Sport Psychology, 8, 60-75. https://doi.org/10.1080/ 10413209608406308 . 